\title{
Unified Theory of Electrons and Photons in Heavy Ion Collisions*
}

\author{
Johannes Kirsch, Berndt Müller, and Walter Greiner \\ Institut für Theoretische Physik der Johann-Wolfgang-Goethe Universität, \\ Frankfurt am Main, Germany
}

Z. Naturforsch. 35 a, 579-589 (1980); received March 11, 1980

We present a unified formulation of the interaction of electrons with the electromagnetic field in heavy ion collisions, based on quantized interacting fields. This reduces the effort in treating many-electron systems substantially, as compared with the usual $S$-matrix theory. Both formalisms are shown to be equivalent. The simplification achieved by our new approach is demonstrated in detail for the example of quasi-molecular radiation.

\section{Introduction}

Heavy ion collisions allow to study the behaviour of electrons in strong electric and magnetic fields. When the ions collide at energies near the Coulomb barrier they may come so close that for a short time the surrounding electrons feel the potential of both nuclei (one speaks of a superheavy quasimolecule). For very small distances between the nuclei, superheavy quasiatoms are formed with charge equal to the sum of projectile and target charge. The electrons in this potential more or less follow the so-called quasimolecular orbitals (in short: MO's). However, the collision evolves rapidly and this variation in time causes electron excitations and creation of vacancies which may decay by X-ray emission.

The investigation of vacancy creation processes and quasimolecular X-ray spectra is motivated by the large electron binding energies occurring in the collision of very heavy nuclei. E.g. for the charge of the united atom $Z_{\mathrm{UA}}>172$ the $1 \mathrm{~s}$-level is predicted to join the negative energy continuum, a process which is of fundamental importance because it leads to the decay of the neutral vacuum of the electron-positron field. In the so-called overcritical fields only a charged vacuum state is stable [1].

For the spectroscopy of such strongly bound electron states mainly four experimental methods are presently in use: The measurement of the

\footnotetext{
* Work supported by the Bundesministerium für Forschung und Technologie (BMFT) and by the Gesellschaft für Schwerionenforschung (GSI).

Reprint requests to Prof. Dr. W. Greiner, Robert-MayerStraße 8-10, D-6000 Frankfurt (Main) 1.
}

positron spectra, the vacancy-production probability as function of the impact parameter, the delta-electron cross-section and the MO X-ray anisotropies. In order to derive valid theoretical predictions for these quantities one has to take into account the many-body character of the electronic system. In the present literature it is usual to expand the state vector into configurations. This leads to a system of coupled channels which in the case of many electrons is not solvable without strong restrictions or simplifying assumptions. We have therefore attempted to find a different formulation avoiding the explicit use of configurations. We will show in this paper that it is possible to express all physically relevant quantities in terms of single-particle amplitudes. Our new formalism is based on the extensive use of fieldoperator-techniques. It is equivalent to, but more advantageous than, the standard many-body $S$ matrix approach.

Our paper is organized as follows: In Chapter 2 we put forward the theory of electronic excitations and calculate the hole probabilities as a first application. The main aim of this work, i.e. to develop an expression for the quasimolecular radiation amplitude and to discuss in detail its application to the X-ray anisotropy, is presented in chapter 3 . The appendices contain a short survey of the $S$ matrix formalism and the main steps of the proof that the two theories are equivalent.

\section{Field Theoretical Description}

\subsection{The field equations}

We start by writing down the Lagrangian for the total system of electrons and nuclei. In order 
to avoid possible complications due to nuclear reactions we limit ourselves to scattering processes below the Coulomb barrier. We may then treat the nuclei as elementary particles of the according field operators $\Phi_{K}^{*}, \Phi_{K}(K=1,2)$ creating or annihilating nuclei with mass $M_{K}$ and charge $Z_{K}$. Under these assumptions the Lagrangian has the form:

$$
\begin{aligned}
\mathscr{L}= & \bar{\Phi}_{\mathrm{e}}\left(i \gamma^{\mu} \partial_{\mu}-m_{\mathrm{e}}\right) \Phi_{\mathrm{e}}+\mathscr{L}_{\mathrm{N}} \\
& +\frac{1}{2}\left(\partial_{\mu} A_{v}\right)\left(\partial^{\mu} A^{v}\right)+\frac{1}{2}\left\{j_{\mu} \mathrm{e}, A^{\mu}\right\} \\
& +\left(j_{\mu}{ }^{1}+j_{\mu}^{2}\right) A^{\mu} .
\end{aligned}
$$

Here $L_{\mathrm{N}}$ is the nuclear part of the Lagrangian, and $\Phi_{\mathrm{e}}, A_{\mu}$ are the field operators for the electron and photon field, respectively.

$$
j_{\mu} \mathrm{e} \equiv-\frac{e}{2}\left[\bar{\Phi}_{\mathrm{e}}, \gamma_{\mu} \Phi_{\mathrm{e}}\right]
$$

is the electronic current operator, $j_{\mu}{ }^{K}(K=1,2)$ the nuclear currents that will be discussed below. The symmetrization of the interaction term and the antisymmetrization of the electronic current (2.1a) guarantee the charge neutrality of the vacuum [2].

To keep the Lagrangian of the two nuclei, $L_{\mathrm{N}}$, as simple as possible we can make further assumptions. For heavy ions the spin of the nuclei is very small compared to the angular momentum of their motion and may be neglected. Their orbit itself can be described nonrelativistically, because the relative velocity at energies below the Coulomb barrier is small $(v / c \sim 0.1)$. Then we have

$\mathscr{L}_{\mathrm{N}}=\sum_{K=1,2} \Phi_{K} * i \partial_{\mathrm{t}} \Phi_{K}-\frac{1}{2 M_{K}}\left(\nabla \Phi_{K} *\right) \cdot\left(\nabla \Phi_{K}\right)$

whence it follows by minimal coupling:

$$
\begin{gathered}
j_{\mu}{ }^{K}=Z_{K} e\left(\Phi_{K} * \Phi_{K}, \frac{i}{2 M_{K}}\left[\Phi_{K} \nabla \Phi_{K} *-\Phi_{K} * \nabla \Phi_{K}\right]\right. \\
\left.-\frac{Z_{K} e}{M_{K}} \boldsymbol{A} \Phi_{K} * \Phi_{K}\right) .
\end{gathered}
$$

The field equations are obtained by variation of $\int \mathrm{d}^{4} x \mathscr{L}$ with respect to $\bar{\Phi}_{\mathrm{e}}, \Phi_{1} *, \Phi_{2}{ }^{*}$ and $A_{\mu}$

$$
\begin{gathered}
\left(i \gamma^{\mu} \partial_{\mu}-m_{\mathrm{e}}\right) \Phi_{\mathrm{e}}=\frac{e}{2}\left\{\gamma_{\mu} \Phi_{\mathrm{e}}, A^{\mu}\right\} \\
{\left[\frac{1}{2 M_{K}}\left(-i \boldsymbol{\nabla}-Z_{K} e \boldsymbol{A}\right)^{2}+Z_{K} e A_{0}\right] \Phi_{K}=i \partial_{\mathrm{t}} \Phi_{K},}
\end{gathered}
$$

$$
\square A_{\mu}=j_{\mu} \mathrm{e}+j_{\mu}{ }^{1}+j_{\mu}^{2} .
$$

First of all we investigate the solution of the last eq. (2.5), which is formally given by

$$
A_{\mu}=A_{\mu}^{(+)}+A_{\mu}^{\mathrm{e}}+A_{\mu}^{1}+A_{\mu}^{2}
$$

with

$$
\begin{aligned}
& \square A_{\mu}^{(+)}(x)=0, \\
& A_{\mu} \mathrm{e}^{\mathrm{e}}(x)=\int \mathrm{d}^{4} y D^{\operatorname{Ret}}(x-y) j_{\mu} \mathrm{e}(y), \\
& A_{\mu}{ }^{K}(x)=\int \mathrm{d}^{4} y D^{\operatorname{Ret}}(x-y) j_{\mu}{ }^{K}(y), \\
& K=1,2
\end{aligned}
$$

where $D^{\text {Ret }}(x-y)$ is the retarded photon propagator. $A_{\mu}{ }^{(+)}$is the free photon in-field, $A_{\mu}{ }^{\mathrm{e}}, A_{\mu}{ }^{K}$ are the retarded electromagnetic fields produced by the electrons and the nuclei, respectively.

Note that the integrals in $(2.6 \mathrm{a}, \mathrm{b})$ do not in general exist as they stand. In order to ensure their existence the currents have to be modified, e.g. they must be adiabatically switched on and off. This can be done by the substitution [3]

$$
j_{\mu} \rightarrow j_{\mu} e^{-\alpha|t|}, \quad \alpha>0,
$$

where at the end of all calculations we take the limit $\alpha \rightarrow 0$.

To solve the first two field equations (2.3), (2.4) we neglect the backreaction of the electrons onto the nuclear current, which is allowed for heavy systems, the selfinteraction of each individual nucleus and their interaction with the free radiation field. In this way we can treat the nuclear motion independent of the electronic system and obtain :

$\left[\frac{1}{2 M_{1}}\left(-i \boldsymbol{\nabla}-Z_{1} e \boldsymbol{A}_{2}\right)^{2}+Z_{1} e A_{2}{ }^{0}\right] \Phi_{1}=i \partial_{t} \Phi_{1}$,

and a like equation with the indices 1, 2 exchanged.

It is customary to replace (2.7) by its classical analogue i.e. to assume that the nuclei move along classical trajectories. The electrons are influenced by the nuclear motion via the nuclear currents which in this approximation are given by

$$
j_{\mu}^{K}=Z_{K} e \delta\left(\boldsymbol{x}-\boldsymbol{R}_{K}(t)\right)\left(1, \boldsymbol{v}_{K}\right) .
$$

\subsection{Solution of the equation for the electron field}

In the following we neglect the influence of the free electromagnetic in-field $A_{\mu}^{(+)}$. This amounts to the assumption that there are no incoming 
photons, i.e. no outside radiation impinging on the quasimolecule, and to the neglect of the zeropoint fluctuations of the electromagnetic field that are responsible, e.g., for the largest part of the Lamb shift. We do not, however, neglect electronic bremsstrahlung. Then the field equation for $\Phi_{\mathrm{e}}(2.3)$ takes the form:

$$
\hat{\mathrm{d}} \Phi_{\mathrm{e}}=\frac{e}{2}\left\{\gamma^{\mu} \Phi_{\mathrm{e}}, A_{\mu} \mathrm{e}\right\}
$$

with the notation

$$
\hat{\mathrm{d}} \equiv i \gamma^{\mu}\left[\partial_{\mu}+i e\left(A_{\mu}^{1}+A_{\mu}^{2}\right)\right]-m_{\mathrm{e}} .
$$

In order to solve this equation we make a Fock decomposition of the field operator $\Phi_{\mathrm{e}}$.

$$
\Phi_{\mathrm{e}}(x)=\sum_{n} a_{n}(t) \Psi_{n}(x)
$$

with respect to a complete, orthonormal set of single particle wavefunctions $\left\{\Psi_{n}\right\}$, to be specified below. From the equal time commutation relation

$$
\left\{\Phi_{\mathrm{e}}{ }^{+}(x), \Phi_{\mathrm{e}}\left(x^{\prime}\right)\right\}_{t=t^{\prime}}=\delta\left(\boldsymbol{x}-\boldsymbol{x}^{\prime}\right)
$$

follows immediately

$$
\left\{a_{n}^{+}(t), a_{m}(t)\right\}=\delta_{n m} .
$$

If we assume that for $t \rightarrow-\infty$ the functions $\Psi_{n}$ go over into the electronic eigenstates of the system, the ground state may be constructed by defining a Fermi surface such that

$$
\begin{array}{rlr}
\stackrel{\circ}{a}_{n}|F\rangle & =0, & n>F, \\
\stackrel{\circ}{a}_{n}+|F\rangle & =0, & n<F,
\end{array}
$$

where

$$
\stackrel{\circ}{a}_{n}=\lim _{t \rightarrow-\infty} a_{n}(t) .
$$

The limiting procedure in $(2.11 \mathrm{a})$ is justified by the formal trick presented in $(2.6 \mathrm{~d})$. For the same reason the ground state $|F\rangle$ may be assumed to be a slater determinant. We also like to note that in a realistic system we deal with an ensemble of different pre-ionized atoms that must be described by a density matrix. Then the expectation value of any operator $\hat{O}$ is given by

$$
\langle\hat{O}\rangle=\sum_{F} W_{F}\langle F|\hat{O}| F\rangle,
$$

where $W_{F}$ is the statistical weight of a pure state $|F\rangle$. Equation (2.11) completely specifies the boundary condition of the electronic system before the collision. Now we insert the expansion (2.9) into (2.8) and obtain with the help of $(2.6 \mathrm{~b})$ and (2.1 a):

$$
\begin{aligned}
& \sum_{n} i \gamma_{0} \Psi_{n}(x) \dot{a}_{n}\left(t_{x}\right)+\left(\hat{\mathrm{d}} \Psi_{n}(x)\right) a_{n}\left(t_{x}\right) \\
= & -\frac{1}{4} e^{2} \gamma^{\mu} \int \mathrm{d}^{4} y D^{\mathrm{Ret}}(x-y) \\
& \cdot \sum_{l m n} \bar{\Psi}_{l}(y) \gamma_{\mu} \Psi_{m}(y) \Psi_{n}(x) \\
& \cdot\left[a_{n}\left(t_{x}\right) a_{l}+\left(t_{y}\right) a_{m}\left(t_{y}\right)\right. \\
& \quad-a_{n}\left(t_{x}\right) a_{m}\left(t_{y}\right) a_{l}{ }^{+}\left(t_{y}\right) \\
& +a_{l}+\left(t_{y}\right) a_{m}\left(t_{y}\right) a_{n}\left(t_{x}\right) \\
& \left.-a_{m}\left(t_{y}\right) a_{l}+\left(t_{y}\right) a_{n}\left(t_{x}\right)\right]
\end{aligned}
$$

Up to now we have not specified the basis set $\left\{\Psi_{n}\right\}$, which can be done in many ways. For instance we obtain the time-dependent Hartree-Fock equations if we neglect the time dependence of the operators $a_{n}$, taking $a_{n}(t)=a_{n}^{\circ}$, and demand that (2.12) is exact for all matrix elements between states differing only by single particle excitations:

$$
\begin{aligned}
\hat{\mathrm{d}} \Psi_{n}(x)= & -\frac{e^{2}}{4} \gamma^{\mu} \int \mathrm{d}^{4} y D^{\mathrm{Ret}}(x-y) \\
& \cdot \tilde{\sum}_{m}\left(\bar{\Psi}_{m}(y) \gamma_{\mu} \Psi_{m}(y) \Psi_{n}(x)\right. \\
& \left.-\bar{\Psi}_{m}(y) \gamma_{\mu} \Psi_{m}(x) \Psi_{n}(y)\right),
\end{aligned}
$$

where

$$
\widetilde{\sum_{m}} \equiv \frac{1}{2}\left(\sum_{m<F}-\sum_{m>F}\right) \cdot
$$

Let us consider the tilde sum which has, by definition, the property

$$
\begin{aligned}
\sum_{m} & =\sum_{F_{0}<m<F}+\frac{1}{2}\left(\sum_{m<F_{0}}-\sum_{m>F_{0}}\right) \\
& \equiv \sum_{m}+\sum_{m}^{\mathrm{HF}},
\end{aligned}
$$

where $F_{0}$ is the Fermi surface of a system of two fully ionized atoms. It means that the HartreeFock sum $\sum_{m}^{\mathrm{HF}}$ is the sum over all real occupied states, while the remainder, the Uehling sum $\sum_{m}^{\text {Uh }}$, describes the effects of vacuum polarization.

Another possibility to choose the basis is to take the solutions of

$$
\hat{\mathrm{d}} \Psi_{n}(x)=0 .
$$

Then there remains a rather complicated time dependence for the $a_{n}$ 's. Since we wish to work in the following with this bases we are led to a nonlinear equation which cannot, of course, be solved 
exactly. A perturbative treatment yields, e.g., in first order

$$
\begin{aligned}
a_{K}^{(1)}(t)= & a_{K}^{(0)}-\frac{i e^{2}}{2} \gamma^{0} \gamma^{\mu} \int \mathrm{d}^{4} y \sum_{l m n} \Psi_{l}(y) \gamma_{\mu} \Psi_{m}(y) \\
& \cdot \int_{-\infty}^{t} \mathrm{~d} \tau\left\langle\Psi_{K}(x)\left|D^{\operatorname{Ret}}(x-y)\right| \Psi_{n}(x)\right\rangle \quad(2.14 \\
& \cdot\left(2 a_{l}^{(0)} a_{m}^{(0)} a_{n}^{(0)}+\delta_{l n} a_{m}^{(0)}-\delta_{l m} a_{n}^{(0)}\right),
\end{aligned}
$$

where the zeroth order solutions $a_{n}{ }^{(0)}$ are identical with the $\stackrel{\circ}{a}_{n}$ 's defining the ground state $(2.11)$. As we are interested mainly in the behaviour of the inner shells we shall neglect the electron-electron interaction, the most important influence of which will be an energy shift of the MO's. Then, as an immediate consequence, the $a_{n}$ 's are constant in time. That this is an adequate procedure has been shown by Rihan et al. [4]. Because $\hat{d}$ is a self-adjoint operator, the solutions of (2.14) form a complete and orthonormal basis for all times, if they did so at $t \rightarrow-\infty$. They are usually obtained by first solving the stationary two center Dirac equation [5]:

$$
\begin{aligned}
& \boldsymbol{\gamma}^{0}\left[\boldsymbol{\gamma} \cdot \boldsymbol{p}+m_{\mathrm{e}}+\gamma^{0} V_{\mathrm{TC}}(\boldsymbol{r}, R(t))\right] \varphi_{n}(\boldsymbol{r}, R(t)) \\
& =\omega_{n}(R(t)) \varphi_{n}(\boldsymbol{r}, R(t))
\end{aligned}
$$

where

$$
\begin{aligned}
& V_{\mathrm{TC}}(\boldsymbol{r}, R(t))=-\frac{Z_{1} e^{2}}{\left|\boldsymbol{r}-\frac{M_{2}}{M_{1}+M_{2}} R \boldsymbol{e}_{z}\right|} \\
& -\frac{Z_{2} e^{2}}{\left|\boldsymbol{r}+\frac{M_{1}}{M_{1}+M_{2}} R \boldsymbol{e}_{z}\right|}
\end{aligned}
$$

The nuclear electromagnetic field is represented in the Coulomb gauge. By the choice of the two center Coulomb potential (2.15a) we have fixed our coordinate system such that the $z$-axis coincides with the connection line of the two nuclei. The expansion of the $\Psi_{n}$ 's :

$\Psi_{n}^{(+)}(\boldsymbol{r}, t)=\sum_{l} b_{n l}^{(+)}(t) \varphi_{l}(\boldsymbol{r}, R(t)) \exp \left\{-i \int^{t} t d^{\prime} \omega_{l}\right\}$

is inserted into (2.14) and we obtain by projecting at every instant of time onto the stationary basis $\varphi_{n}$ a system of coupled equations for the amplitudes $b_{n l}^{(+)}$:

$$
\begin{aligned}
\dot{b}_{n l}^{(+)}= & -\sum_{l^{\prime}} b_{n l^{\prime}}^{(+)} \exp \left\{i \int^{t} \mathrm{~d} t^{\prime}\left(\omega_{l}-\omega_{l^{\prime}}\right)\right\} \\
& \cdot\left\langle\varphi_{\mathrm{e}}\left|\partial_{t}-i \mathrm{e} \gamma^{0} \gamma \cdot\left(\boldsymbol{A}^{1}+\boldsymbol{A}^{2}\right)\right| \varphi_{l^{\prime}}\right\rangle,
\end{aligned}
$$

where the index $n$ and the $(+)$-sign characterize the boundary condition:

$$
\lim _{t \rightarrow-\infty} b_{n l}^{(+)}(t)=\delta_{n l} .
$$

Of course, the operators $a_{n}$ depend on the choice of the boundary condition. We emphasize this by the notation $a_{n}^{(+)}$. Although the use of the two center distance $R(t)$ as scattering coordinate allows for a simple description of the nuclear motion, one must keep in mind that it leads to unphysical couplings in the asymptotic regions. Therefore, a correct treatment must take into account the translation of the electrons with the binding nuclei [6]. A simply way to include this effect is to rotate the basis of stationary states $\varphi_{l}$ by a suitable unitary transformation (the so-called translation "factors"). As a result the coupling matrix elements in (2.17) are slightly modified.

\subsection{Vacancy creation rates}

As a first application of the theory we want to calculate the final vacancy probability of an electronic state $k$ with $k<F$. In order to do so we note that the field operator $\Phi_{\mathrm{e}}$ can be also expanded in the basis $\left\{\Psi_{n}^{(-)}\right\}$of single-particle states which go over into eigenstates of the ions long after the collision. For these states an expansion like (2.16) satisfies the boundary conditions

$$
\lim _{t \rightarrow+\infty} b_{n l}^{(-)}(t)=\delta_{n l} \text {. }
$$

Equating the two different Fock expansions

$$
\sum_{n} a_{n}^{(+)} \Psi_{n}^{(+)}=\Phi_{\mathrm{e}}=\sum_{n} a_{n}^{(-)} \Psi_{n}^{(-)}
$$

we obtain a connection between the corresponding particle operators:

$$
a_{n}^{(-)}=\sum_{m} a_{m}^{(+)}\left\langle\Psi_{n}^{(-)} \mid \Psi_{m}^{(+)}\right\rangle .
$$

The $S$-matrix element $\left\langle\Psi_{n}^{(-)} \mid \Psi_{m}^{(+)}\right\rangle$is time independent and can be expressed by the occupation amplitudes. To show this we employ the time evolution operator $U\left(t, t^{\prime}\right)$ satisfying

$$
\hat{d}_{t} U\left(t, t^{\prime}\right)=0 \text {. }
$$

Then

$$
\begin{aligned}
& \left\langle\Psi_{n}^{(-)}(t) \mid \Psi_{m}^{(+)}(t)\right\rangle=\lim _{\tau \rightarrow \infty}\left\langle\varphi_{n}(\tau)\right. \\
& \cdot \exp \left\{-i \int^{\tau} \mathrm{d} \tau^{\prime} \omega_{n}\right\}\left|U^{+}(t, \tau) \Psi_{m}^{(+)}(t)\right\rangle \\
& =\lim _{\tau \rightarrow \infty}\left\langle\varphi_{n}(\tau) \exp \left\{-i \int^{\tau} \mathrm{d} \tau^{\prime} \omega_{n}\right\} \mid \Psi_{m}^{(+)}(\tau)\right\rangle \\
& =b_{m n}^{(+)}(\infty)
\end{aligned}
$$


holds. The replacement of the true outgoing wave $\Psi_{n}$ by the stationary solution $\varphi_{n}$ is justified by the adiabatic hypothesis, $(2.6 \mathrm{~d})$, that the limiting procedure $t \rightarrow+\infty$ exists. Therefore we may write

$$
a_{n}^{(-)}=\sum_{m} a_{m}^{(+)} b_{m n}^{(+)}(\infty) .
$$

Then the final hole probability in state $K$ is given by

$$
P_{K}=1-\left\langle F\left|a_{K}^{(-)}+a_{K}^{(-)}\right| F\right\rangle .
$$

The ground state expectation value can be evaluated by expressing the operator $a_{K}^{(-)}$in terms of $a_{m}^{(+)}$ via (2.19). In case that the $a_{m}^{(+)}$change with time, everything must be expressed in terms of $a_{m}^{(+)}$ $(-\infty)=a_{m}$ (see Eq. (2.14a)). Making use of the definition of the Heisenberg state $|F\rangle,(2.11)$, we obtain finally

$$
P_{K}=\sum_{m>F}\left|b_{m K}^{(+)}(\infty)\right|^{2} .
$$

By a comparison with the standard method [7] to derive (2.20) we can see the advantage of our formalism. A further and more striking example for the superiority of the operator formalism and the employment of the Heisenberg picture is provided by the derivation of the quasimolecular radiation cross-section. This will be given in the next section.

\section{The Quasimolecular Radiation Cross Section}

\subsection{Motivation}

One of the effects reflecting the quasimolecular binding energies seems to be the MO X-ray anisotropy [8]

$$
\eta(\omega)=\frac{\mathrm{d} \sigma\left(\vartheta=90^{\circ}\right)-\mathrm{d} \sigma\left(\vartheta=0^{\circ}\right)}{\mathrm{d} \sigma\left(\vartheta=0^{\circ}\right)},
$$

where $\vartheta$ is the angle between the photon detection direction and the beam axis. Whereas it is not obvious how to extract information about the large binding energy of the $1 \mathrm{~s}$ level at the united atom limit from the measured MO X-ray spectra (they do not seem to exhibit any cut-off at the united atom $K_{\alpha}$-line due to dynamical broadening), the anisotropy is experimentally known to depend sensitively on the photon frequency and to peak at the binding energy in the united atom [9]. In other words, it seems that precisely this quantity allows for a spectroscopy of intermediate electronic levels. This has been anticipated on the basis of simple theoretical models $[10-13]$, and has been extensively and convincingly verified in experiments by Wölfli and his collaborators [14].

Supposedly, the peak in the anisotropy is caused by the strong rotational coupling between the levels at close distances reflecting the fact that the electron cannot follow the swift rotation of the internuclear axis [15]. This is the so-called slippageeffect. Müller and Greiner [8] have shown that the anisotropy depends critically on the population of the various magnetic substates, especially in the L-shell of the united atom. Any attempt to calculate the anisotropy ab initio, i.e. without ad hoc introducing an alignment, must treat all levels of a subshell in the same manner. The simplest case allowing for excitation of vacancies and for the formation of an alignment is a model including the $\mathrm{K}, \mathrm{L}$ and $\mathrm{M}$ shells, where the $\mathrm{K}$ and $\mathrm{L}$ shells are assumed to be initially filled and the $\mathrm{M}$ shell to be vacant. Working with configurations, we then would have to solve a system about 40000 coupled channels (see Eq. (B6) in the appendix). Even if this could be circumvented, the usual $S$-matrix approach would require the summation of a similar number of final channels in order to obtain the cross-section. We shall see that, with the help of the formalism developed in the previous section, we are able to calculate the radiation cross-section much more easily.

We start by re-stating the basic equations.

$$
\begin{aligned}
\square A_{\mu}(x) & =j_{\mu}(x)=-\frac{e}{2}\left[\bar{\Phi}(x), \gamma_{\mu} \Phi(x)\right], \\
\Phi(x) & =\sum_{n} a_{n} \Psi_{n}(x), \\
\hat{d} \Psi_{n}(x) & =0 .
\end{aligned}
$$

Here we have suppressed the index e denoting the electronic operators. We also have not specified the boundary conditions for the wave functions $\Psi_{n}$, but it will be understood in the following that they are of the $(+)$-type, eqs. $(2.16-17)$. We further remind the reader that with electron-electron correlations neglected the particle operators $a_{n}, a_{n}{ }^{+}$ are constant in time, i.e. $a_{n}(t)=\stackrel{\circ}{a}_{n}$.

The differential radiation cross-section from which we determine the anisotropy (3.1) is given by

$$
\frac{\mathrm{d} \sigma}{\mathrm{d} \Omega_{\mathrm{S}}}=\left(\frac{\mathrm{d} \sigma}{\mathrm{d} \Omega_{\mathrm{S}}}\right)_{\mathrm{SCATT}} \mathrm{d}^{3} k \sum_{\lambda} I(\boldsymbol{k}, \lambda) .
$$


In the classical approximation the scattering crosssection may be replaced by the Rutherford crosssection. All we need to know now is the intensity $I(\boldsymbol{k}, \lambda)$ of photons with wavevector $\boldsymbol{k}$ and polarization $\lambda . I(\boldsymbol{k}, \lambda)$ can be expressed as the ground state expectation value of the corresponding photon number operator, i.e.

$$
I(\boldsymbol{k}, \lambda)=\left\langle F\left|\mathrm{~d}_{\boldsymbol{k} \lambda}^{(-)} \dagger \mathrm{d}_{\boldsymbol{k} \lambda}^{(-)}\right| F\right\rangle,
$$

where $\mathrm{d}_{\boldsymbol{k} \boldsymbol{\lambda}}^{(-)} \dagger$ and $\mathrm{d}_{\boldsymbol{k} \boldsymbol{\lambda}}^{(-)}$are the creation and annihilation operators of the asymptotic photon field $(t \rightarrow+\infty)$, in analogy with the calculation of the hole probabilities at the end of the last section.

\subsection{Solution of the equation for the photon field}

The formal solution of (3.2) is given by a superposition of the free in-field and the field produced by the electrons:

$$
\begin{aligned}
A_{\mu}(x)= & A_{\mu}^{(+)}(x) \\
& +\int \mathrm{d}^{4} y D^{\operatorname{Ret}}(x-y) j_{\mu}(y) .
\end{aligned}
$$

It involves the boundary condition

$$
\lim _{t \rightarrow-\infty} A_{\mu}(x)=\lim _{t \rightarrow-\infty} A_{\mu}^{(+)}(x)
$$

because the retarded propagator vanishes inside the backward light cone.

We shall now exploit the freedom inherent in the gauge invariance of the electromagnetic field by imposing a special condition on $A_{\mu}$. As usual one shows [16] that the condition

$$
\left(\partial_{\mu} A^{\mu}\right)_{\text {pos. }}|F\rangle=0
$$

involving the positive frequency part of $\partial_{\mu} A^{\mu}$ implies the cancellation of the contribution from longitudinal and scalar photons. It is therefore sufficient to sum over the transverse polarization $\lambda=1,2$ in (3.5). Since we are interested in the number of photons long after the collision we have to investigate the radiation field in the asymptotic region $t \rightarrow+\infty$. Therefore we define a free outfield by

$$
\lim _{t \rightarrow+\infty} A_{\mu}^{(-)}(x)=\lim _{t \rightarrow+\infty} A_{\mu}(x) .
$$

We now apply the relation between the retarded and advanced propagator

$$
D^{\operatorname{Ret}}(x-y)=D^{\operatorname{Adv}}(x-y)-\Delta_{0}(x-y),
$$

$\Delta_{0}$ being the Schwinger function, and obtain

$$
A_{\mu}^{(-)}(x)=A_{\mu}^{(+)}(x)-\int \mathrm{d}^{4} y \Delta_{0}(x-y) j_{\mu}(y)
$$

because $D^{\mathbf{A d v}}(x)$ vanishes inside the forward light cone.

$A_{\mu}^{(-)}$satisfies the free Maxwell equation, since $\square x \Delta_{0}(x-y)=0$. Being solutions of the homogeneous wave equation, the asymptotic fields $A_{\mu}^{( \pm)}$ may be quantized in the standard way and can be expanded in terms of photon operators:

$$
\begin{aligned}
A_{\mu}^{( \pm)}(x)= & \sum_{\boldsymbol{k}, \lambda} d_{\boldsymbol{k} \lambda}^{( \pm)} q_{\boldsymbol{k} \lambda \mu}(x) \\
& +d_{\boldsymbol{k} \lambda}^{( \pm)}{ }^{\dagger} q_{\boldsymbol{k} \lambda \mu}^{*}(x)
\end{aligned}
$$

with

$$
\left[d_{\boldsymbol{k} \lambda}^{( \pm)}, d_{\boldsymbol{k} \lambda}^{( \pm) \dagger}\right]=\delta_{\boldsymbol{k} \boldsymbol{k}^{\prime}}, \delta_{\lambda \lambda^{\prime}}
$$

and

$$
q_{\boldsymbol{k} \lambda \mu}(x)=\frac{1}{V^{1 / 2}} \frac{1}{(2 \omega)^{1 / 2}} \varepsilon_{\boldsymbol{k} \lambda \mu} e^{-i k x} .
$$

$\lambda$ counts the polarization, $\mu$ denotes the four-vector index. The basis functions $q_{\boldsymbol{k}, \mu}$ obey the orthogonality relation

$$
i \int_{V} d^{3} x q_{\boldsymbol{k}^{\prime} \lambda^{\prime} \mu}^{*}(x) \stackrel{\leftrightarrow}{\partial_{0}} q_{\boldsymbol{k} \lambda}^{\mu}(x)=\delta_{\boldsymbol{k} \boldsymbol{k}^{\prime}} \delta_{\lambda \lambda^{\prime}}
$$

with the abbreviation

$$
A \stackrel{\leftrightarrow}{\partial_{0}} B \equiv A\left(\partial_{0} B\right)-\left(\partial_{0} A\right) B
$$

Equation (3.8) induces a relation between the operators for in- and out-photons which is obtained by projecting out a specific state $q_{\boldsymbol{k}, \mu}$ :

$$
d_{\boldsymbol{k} \lambda}^{(-)}=d_{\boldsymbol{k} \lambda}^{(+)}-i h_{\boldsymbol{k} \lambda}
$$

with

$$
\begin{aligned}
h_{\boldsymbol{k} \lambda}=\int_{V} \mathrm{~d}^{3} x q_{\boldsymbol{k} \lambda \mu}^{*}(x) \\
\quad \cdot \partial_{0} \int \mathrm{d}^{4} y \Delta_{0}(x-y) j^{\mu}(y) .
\end{aligned}
$$

\subsection{The radiation intensity}

Again we make the assumption that the initial state does not contain free photons. The Heisenberg state of our system is then defined by

$$
\mathrm{d}_{\boldsymbol{k} \lambda}^{(+)}|F\rangle=0
$$

for all $\boldsymbol{k}, \lambda$. Then we obtain from (3.6), (3.10) and (3.11) for the intensity:

$$
I(\boldsymbol{k}, \lambda)=\left\langle F\left|h_{\boldsymbol{k} \lambda}^{* \dagger} h_{\boldsymbol{k}\rangle}\right| F\right\rangle
$$

The properties of the Schwinger function may be utilized to get a simplified expression for $h_{\boldsymbol{k} \lambda}$ :

$$
h_{\boldsymbol{k} \lambda}=\int \mathrm{d}^{4} x q_{\boldsymbol{k} \lambda \mu}^{*}(x) j^{\mu}(x)
$$

By expanding $j^{\mu}(x)$ in terms of electron creation and annihilation operators and using the definition 
of the Hartree-Fock sum $(2.13 \mathrm{~b})$ we finally obtain

$$
I(\boldsymbol{k}, \lambda)=\left|\sum_{n}^{\mathrm{HF}} H_{\boldsymbol{k} \lambda}^{n n}\right|^{2}+\sum_{\substack{m<F \\ n>F}}\left|H_{\boldsymbol{k} \lambda}^{n m}\right|^{2}
$$

with the amplitudes

$$
\begin{aligned}
H_{\boldsymbol{k} \lambda}^{n m}= & -e \int \mathrm{d} t e^{i \omega t} \\
& \cdot\left\langle\Psi_{n}\left|\frac{1}{(V 2 \omega)^{1^{\prime 2}}} \varepsilon_{\boldsymbol{k} \lambda \mu} e^{-i \boldsymbol{k x}} \gamma^{\mu}\right| \Psi_{m}\right\rangle .
\end{aligned}
$$

For the details concerning the derivation of (3.13) and (3.14) we refer to appendix $A$.

The practicability of (3.14) becomes obvious if we try to calculate the anisotropy on the basis of the model with initially full $\mathrm{K}$ - and L-shells and empty M-shell discussed at the begin of this chapter. It means that the system is restricted now on 10 electrons and 18 basis states. Then a set of 18 coupled equations (Eq. (2.17)) has to be solved (for 18 boundary conditions) and both double sums in (3.14) run only over a small range $(n, m \leqq 18)$. On the other hand we have eq. (B6), a system of $\left(\begin{array}{l}18 \\ 10\end{array}\right)$ coupled configurations (to solve for just as many boundary conditions!) and the corresponding summation in the cross-section. The reason for the great simplification achieved by (3.14) is the use of the Heisenberg picture together with the Fock representation. The disadvantage of the $S$-matrix formalism is due to the fact that one gets much more information than necessary. First we have to determine the amplitude for each channel and then to sum over all channels. In the field operator approach one calculates only the quantity of interest and nothing more. We conclude this section with some remarks about coincidence spectra produced by simultaneously measuring a quasimolecular photon $(\boldsymbol{k}, \lambda)$ and a characteristic K X-ray from the single atom. For instance, a radiative MO-transition from $2 p_{1 / 2} \sigma$ to $1 \mathrm{~s} \sigma$ creates a vacancy in the $2 p_{1 / 2} \sigma$-level which will be transferred into the $\mathrm{K}$-shell of the lighter collision partner, where it decays radiatively. Therefore we have to calculate the expectation value of the product of the number operators for quasimolecular photons and final $K$ vacancies:

$$
I(\boldsymbol{k}, \lambda, K)=\left\langle F\left|\mathrm{~d}_{\boldsymbol{k} \lambda}^{(-)+} \mathrm{d}_{\boldsymbol{k} \lambda}^{(-)} a_{\boldsymbol{K}}^{(-)} a_{\boldsymbol{K}}^{(-)+}\right| F\right\rangle .
$$

Utilizing (3.10), (2.19) and neglecting all effects from vacuum polarization and the positron states we are led to

$$
\begin{aligned}
I(\boldsymbol{k}, \lambda, K)= & \sum_{\substack{i j n \\
m s t}} b_{s K}(\infty) b_{t K}^{*}(\infty) H_{\boldsymbol{k} \lambda}^{i j *} H_{\boldsymbol{k} \lambda}^{n m} \\
& \cdot\left\langle F\left|a_{j}{ }^{+} a_{i} a_{n}{ }^{+} a_{m} a_{s} a_{t^{+}}\right| F\right\rangle .
\end{aligned}
$$

After evaluation of the matrix element in (3.15) we obtain six terms which can be easily computed once $b_{n l}(\infty)$ and $H_{\boldsymbol{k} \lambda}^{n m}$ are known. We note that a more detailed specification of the final state does not require more complicated dynamical calculations, i.e. the simplicity of our treatment remains.

\section{A Schematic Model}

Finally we want to investigate the radiation formula (3.14) with respect to the temporal order of excitation and radiation. For that purpose we consider a one-electron model which enables us to compare all results of our approach with the according results of the S-matrix formalism, eqs. (B 1), (B 7). Suppressing photon quantum numbers the radiation intensity can be expressed in the two equivalent ways:

$$
I=\sum_{n>F_{0}}\left|c_{n 1}\right|^{2}=\sum_{n>F_{0}}\left|H_{n 1}\right|^{2}
$$

with

$$
\begin{aligned}
c_{n 1} \cong & \int_{-\infty}^{\infty} \mathrm{d} t e^{i \omega t} \sum_{l, l^{\prime}} b_{n l}^{(-)^{*}} b_{1 l^{\prime}}^{(+)}\left\langle\varphi_{l}\left|\boldsymbol{j} \cdot \boldsymbol{q}^{*}\right| \varphi_{l^{\prime}}\right\rangle \\
& \cdot \exp \left\{i \int^{t} \mathrm{~d} t^{\prime}\left(\omega_{l}-\omega_{l^{\prime}}\right)\right\}, \\
H_{n 1} \cong & \int_{-\infty}^{\infty} \mathrm{d} t e^{i \omega t} \sum_{l, l^{\prime}} b_{n l}^{(+)^{*}} b_{1 l^{\prime}}^{(+)}\left\langle\varphi_{l}\left|\boldsymbol{j} \cdot \boldsymbol{q}^{*}\right| \varphi_{l^{\prime}}\right\rangle \\
& \cdot \exp \left\{i \int^{t} \mathrm{~d} t^{\prime}\left(\omega_{l}-\omega_{l^{\prime}}\right)\right\},
\end{aligned}
$$

where $b_{n l}^{(t)}$ are the solutions of (2.17). Since $q_{\boldsymbol{k} \gg 0}=0$ for transversal photons, (4.3) becomes identical with (3.14a) using the notation $\boldsymbol{j}=-e \boldsymbol{\gamma}$. Furthermore, we assumed the electron to be in the ground state (1). Of course, the ,one“-particle model still suffers from possible manybody effects out of the vacuum, but we shall neglect these contributions in the following.

To study the temporal order of excitant and radiative processes we make an ansatz for the transition matrix elements

$$
\begin{aligned}
\left\langle\varphi_{l}\left|\partial_{t}\right| \varphi_{l^{\prime}}\right\rangle & =\alpha_{l l^{\prime}} \delta\left(t-t_{0}\right), \\
\left\langle\varphi_{l}\left|\boldsymbol{j} \cdot \boldsymbol{q}^{*}\right| \varphi_{l^{\prime}}\right\rangle & =\beta_{l l^{\prime}} \delta\left(t-t_{1}\right) .
\end{aligned}
$$

The $\partial_{t}$-coupling shall be so weak that the excitation may be treated perturbatively. Then we obtain 
in first order

$$
\begin{aligned}
\mathrm{c}_{n 1}^{(1)}= & \mathrm{c}_{n 1}^{(0)}-A_{n 1} \vartheta\left(t_{1}-t_{0}\right) \\
& +B_{n 1} \vartheta\left(t_{0}-t_{1}\right), \\
H_{n 1}^{(1)}= & H_{n 1}^{(0)}-\left(A_{n 1}+B_{n 1}\right) \vartheta\left(t_{1}-t_{0}\right),
\end{aligned}
$$

where

$$
\begin{aligned}
\mathrm{c}_{n 1}^{(0)}= & H_{n 1}^{(0)}=\beta_{n 1} \\
& \cdot \exp \left\{i \int^{t_{1}} \mathrm{~d} t\left(\omega_{n}-\omega_{1}\right)+i \omega t_{1}\right\}, \\
A_{n 1}= & \sum_{l>F_{0}} \beta_{n l} \alpha_{l 1} \exp \left\{i \int^{t_{1}} \mathrm{~d} t\left(\omega_{n}-\omega_{1}\right)\right. \\
& \left.+i \int^{t_{0}} \mathrm{~d} t\left(\omega_{l}-\omega_{1}\right)+i \omega t_{1}\right\} \\
B_{n 1}= & \sum_{l>F_{0}} \beta_{l 1} \alpha_{l n} \exp \left\{i \int^{t_{1}} \mathrm{~d} t\left(\omega_{l}-\omega_{1}\right)\right. \\
& \left.+i \int^{t_{0}} \mathrm{~d} t\left(\omega_{n}-\omega_{l}\right)+i \omega t_{1}\right\}
\end{aligned}
$$

and $\vartheta(t)$ is the unit step function. Equation (4.5) is the analogue to the electron bremsstrahlung occuring in presence of an external field: two terms contribute in first order which correspond to the two possible ways of time-ordering (see Figure 1). Such an interpretation is not possible for $H_{n 1}$ because it is not derived from perturbation theory in the interaction picture.

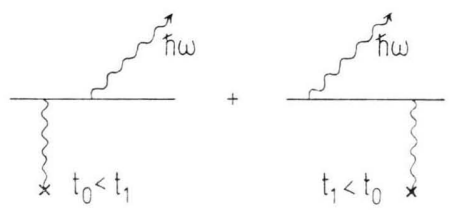

Fig. 1. The electronic bremsstrahlung in an external field.

It is, however, crucial that the intensity does not depend on the use of $c_{n 1}$ or $H_{n 1}$. This is shown for the general case in appendix $B$. For our schematic example it is easy to verify the identity by explicit calculation. Because of

$$
\vartheta\left(t_{0}-t_{1}\right)=1-\vartheta\left(t_{1}-t_{0}\right)
$$

we have

$$
c_{n 1}^{(1)}=H_{n 1}^{(1)}+B_{n 1} .
$$

Neglecting the terms $\sim \alpha_{\ln }^{2}$ we get

$$
I=\sum_{n>F_{0}}\left|c_{n 1}^{(1)}\right|^{2}=\sum_{n>F_{0}}\left|H_{n 1}^{(1)}\right|^{2}+I_{0}
$$

with

$$
I_{0}=\sum_{n>F_{0}} H_{n 1}^{(0)^{*}} B_{n 1}+H_{n 1}^{(0)} B_{n 1}^{*} .
$$

Inserting $(4.7 \mathrm{a}, \mathrm{c})$ we see that $I_{0}$, given by

$$
\begin{aligned}
I_{0}= & \sum_{n, l>F_{0}}\left(\left(\beta_{n 1} \beta_{l 1}^{*} \exp \left\{i \int_{t_{0}}^{t_{1}} \mathrm{~d} t\left(\omega_{n}-\omega_{l}\right)\right\}\right.\right. \\
& \left.+\beta_{n 1}^{*} \beta_{l 1} \exp \left\{-i \int_{t_{0}}^{t_{1}} \mathrm{~d} t\left(\omega_{n}-\omega_{l}\right)\right\}\right) \alpha_{l n},
\end{aligned}
$$

vanishes since the expression in the brackets is symmetric in $n, l$ while $\alpha_{l n}=-\alpha_{n l}$.

\section{Summary and 0utlook}

In this article we have discussed the electronic and radiative part of a heavy ion collision using a quantum field theoretical description. The two basic assumptions made are

(a) the so-called semiclassical approximation, describing the nuclear current classically;

(b) the hypothesis that all interactions may be switched on and off adiabatically.

As an application we investigated the quasimolecular radiation in detail, where further assumptions are made: The most important of them is the neglect of the electron-electron correlations. Because of its simplicity the derived formula allows for the first time to calculate the anisotropy including the many-particle properties of the electronic system. The numerical evaluation of (3.14) is presently carried out for the lead-lead system and will be reported somewhere else in the near future.

\section{Appendix A}

The three-dimensional Fourier representation of Schwinger's delta function for vanishing rest mass is given by

$$
\begin{aligned}
\Delta_{0}(x-y)= & \frac{1}{(2 \pi)^{3}} \int \mathrm{d}^{3} k^{\prime} \frac{\sin \left[\omega_{k^{\prime}}\left(t_{\mathbf{x}}-t_{y}\right)\right]}{\omega_{k^{\prime}}} \\
& \cdot \exp \left\{i \boldsymbol{k}^{\prime} \cdot(\boldsymbol{x}-\boldsymbol{y})\right\},
\end{aligned}
$$

where we use the notation $\omega_{k}=|\boldsymbol{k}|$. Making use of $(3.9 \mathrm{~b})$ we have then

$$
\begin{aligned}
q_{\boldsymbol{k} \lambda \mu}^{*}(x) \mathrm{\partial}_{t_{x}} & \Delta_{0}(x-y) \\
= & q_{\boldsymbol{k} \lambda \mu}^{*}(x)\left(\partial_{t_{x}} \Delta_{0}(x-y)\right) \\
& -\left(\partial_{t_{x}} q_{\boldsymbol{k} \lambda \mu}^{*}(x)\right) \Delta_{0}(x-y) \\
= & q_{\boldsymbol{k} \lambda \mu}^{*}(x) \int \frac{\mathrm{d}^{3} k^{\prime}}{(2 \pi)^{3}} \cos \left[\omega_{k^{\prime}}\left(t_{\mathbf{x}}-t_{y}\right)\right] \exp \left\{i \boldsymbol{k}^{\prime}(\boldsymbol{x}-\boldsymbol{y})\right\} \\
& -i \omega_{k} q_{\boldsymbol{k} \lambda \mu}^{*} \Delta_{0}(x-y) .
\end{aligned}
$$


Therefore $h_{k \lambda}$ consists of two parts

$$
h_{\boldsymbol{k} \lambda}=h_{\boldsymbol{k} \lambda}^{(1)}+h_{\boldsymbol{k} \lambda}^{(2)}
$$

with

$$
\begin{gathered}
h_{\boldsymbol{k} \lambda}^{(1)}=\int_{V} \mathrm{~d}^{3} x q_{\boldsymbol{k} \lambda \mu}^{*}(x) \int \mathrm{d}^{4} y \int \frac{\mathrm{d}^{3} k^{\prime}}{(2 \pi)^{3}} \\
\cdot \cos \left[\omega_{k^{\prime}}\left(t_{\mathrm{x}}-t_{y}\right)\right] \exp \left\{-i \boldsymbol{k}^{\prime}(\boldsymbol{x}-\boldsymbol{y})\right\} j^{\mu}(y), \\
h_{\boldsymbol{k} \lambda}^{(2)}=-\int_{V} \mathrm{~d}^{3} x i \omega_{k} q_{\boldsymbol{k} \lambda \mu}^{*}(x) \\
\cdot \int^{1} \mathrm{~d}^{4} y \Delta_{0}(x-y) j^{\mu}(y) .
\end{gathered}
$$

Now we insert $(3.9 \mathrm{~b})$ into the first part and obtain

$$
\begin{aligned}
& h_{\boldsymbol{k} \lambda}^{(1)}=\frac{1}{V^{1 / 2}} \frac{1}{\left(2 \omega_{k}\right)^{1 / 2}} \cdot \varepsilon_{\boldsymbol{k} \lambda \mu}^{*} \exp \left\{i \omega_{k} t_{\mathrm{x}}\right\} \int \mathrm{d}^{4} y \\
& \cdot \cos \left[\omega_{k}\left(t_{\mathrm{x}}-t_{y}\right)\right] e^{-\boldsymbol{k} \boldsymbol{k} \boldsymbol{y}} j^{\mu}(y) .
\end{aligned}
$$

Similarly $h_{\boldsymbol{k} \lambda}^{(2)}$ is given by

$$
\begin{aligned}
& h_{\boldsymbol{k} \lambda}^{(2)}=\frac{1}{V^{1 / 2}} \frac{1}{\left(2 \omega_{k}\right)^{1 / 2}} \cdot \varepsilon_{\boldsymbol{k} \lambda \mu}^{*} e^{i \omega_{k} t_{x}} \\
& \cdot \int \mathrm{d}^{4} y i \sin \left[\omega_{k}\left(t_{\mathrm{x}}-t_{y}\right)\right] e^{-i \boldsymbol{k} \boldsymbol{y}} j^{\mu}(y) .
\end{aligned}
$$

Adding the two terms we get

$$
\begin{aligned}
& h_{\boldsymbol{k} \lambda}=\frac{1}{V^{1 / 2}} \frac{1}{\left(2 \omega_{k}\right)^{1 / 2}} \cdot \varepsilon_{\boldsymbol{k} \lambda \mu}^{*} e^{i \omega_{k} t_{x}} \int \mathrm{d}^{4} y \\
& \exp \left\{-i \omega_{k}\left(t_{\mathrm{x}}-t_{y}\right)\right\} e^{-i \boldsymbol{k} y} j^{\mu}(y)
\end{aligned}
$$

and finally we obtain (3.13) defining the scalar product as :

$$
\left\langle\Psi_{n}|A| \Psi_{m}\right\rangle=\int \mathrm{d}^{3} x \bar{\Psi}_{n} A \Psi_{m} .
$$

To derive (3.14) we use (2.1 a), (2.9) and (3.14a):

$$
\begin{aligned}
& h_{\boldsymbol{k} \lambda}=-\frac{e}{2} \cdot \int \mathrm{d}^{4} x q_{\boldsymbol{k} \lambda \mu}^{*}(x) \\
& \sum \bar{\Psi}_{n}(x) \gamma_{n, m}^{\mu} \Psi_{m}(x)\left(\mathrm{a}_{n}{ }^{+} \mathrm{a}_{m}-\mathrm{a}_{m} \mathrm{a}_{n}{ }^{+}\right) \\
& =\sum_{n, m} H_{\boldsymbol{k} \lambda}^{n m} a_{n}{ }^{+} a_{m}-\frac{1}{2} \sum_{n} H_{\boldsymbol{k} \lambda}^{n n} .
\end{aligned}
$$

With the notations of $(2.13 \mathrm{a}, \mathrm{b})$ we have

$$
-\frac{1}{2} \sum_{n} H_{\boldsymbol{k} \lambda}^{n n}=-\sum_{n<F_{0}} H_{\boldsymbol{k} \lambda}^{n n}+\sum_{n}^{\mathrm{Uh}} H_{\boldsymbol{k} \lambda}^{n n} .
$$

Since the effects of the vacuum polarisation are expected to be small we shall neglect the Uehling term in the following. Therefore we may write:

$$
\begin{aligned}
I(\boldsymbol{k}, \lambda)= & \sum_{\substack{n, m \\
i, j}} H_{\boldsymbol{k} \lambda}^{i j *} H_{\boldsymbol{k} \lambda}^{n m}\left\langle F\left|a_{j}{ }^{+} a_{i} a_{n}{ }^{+} a_{m}\right| F\right\rangle \\
& -\sum_{\substack{n<F \\
m<F_{0}}} H_{\boldsymbol{k} \lambda}^{n n *} H_{\boldsymbol{k} \lambda}^{m m}-\sum_{\substack{n<F_{0} \\
m<}} H_{\boldsymbol{k} \lambda}^{n n *} H_{\boldsymbol{k} \lambda}^{m m *} \\
& +\sum_{n, m<F_{0}} H_{\boldsymbol{k} \lambda}^{n n^{*}} H_{\boldsymbol{k} \lambda}^{m m} .
\end{aligned}
$$

Evaluating the matrix elements and splitting the $\operatorname{sum} \sum_{n<F}$ in $\sum_{n<F_{0}}+\sum_{n}^{H F}$ we get

$$
\begin{gathered}
I(\boldsymbol{k}, \lambda)=\sum_{\substack{n>F \\
m<\boldsymbol{F}}}\left|H_{\boldsymbol{k} \lambda}^{n m}\right|^{2}+\left(\mid \sum_{n<F_{0}}+\sum_{n}\right]\left[\sum_{m<F_{0}}+\sum_{m}^{\mathrm{HF}}\right] \\
-\left\lfloor\sum_{n<F_{0}}+\sum_{n}^{\mathrm{HF}}\right] \sum_{m<F_{0}}-\sum_{n<F_{0}}\left[\sum_{m<F_{0}}+\sum_{m}^{\mathrm{HF}}\right]+\sum_{\substack{n, m \\
<F_{0}}} j \\
\cdot H_{\boldsymbol{k} \lambda}^{n n *} H_{\boldsymbol{k} \lambda}^{m m},
\end{gathered}
$$

and then the final result (3.14). As mentioned we have worked for the sake of simplicity with the $(+)$-type of $a_{n}$. Of course we are drawn to the same results employing the (-)-type.

\section{Appendix B}

Here we want to demonstrate the equivalence of the formula for $I(\boldsymbol{k}, \lambda)$ obtained from the $S$ matrix formulation of time-dependent perturbation theory and the expression for $I(\boldsymbol{k}, \lambda)$ resulting from our method. In the derivation of Briggs and Dettmann [12] the radiation amplitude is given in first order perturbation theory by

$$
\begin{aligned}
c_{\boldsymbol{k} \lambda}^{f i}= & -i \int_{-\infty}^{\infty} \mathrm{d} t e^{\imath \omega t} \\
& \cdot\left\langle\chi_{f}^{(-)}(t)\left|\boldsymbol{j} \cdot \boldsymbol{q}_{\boldsymbol{k} \lambda}^{*}\right| \chi_{i}^{(+)}(t)\right\rangle .
\end{aligned}
$$

The wave function $\chi_{i}^{(+)}$is a dynamical solution of the many body two center Dirac equation

$$
\sum_{j} \hat{d}_{j} \chi_{i}^{(+)} \equiv\left(i \partial_{t}-H\right) \chi_{i}^{(+)}=0
$$

with the boundary condition

$$
\begin{aligned}
\lim _{t \rightarrow-\infty} \chi_{i}^{(+)}(t)= & \lim _{t \rightarrow-\infty} \Phi_{i}(R(t)) \\
& \cdot \exp \left\{-i \int^{t} \mathrm{~d} t^{\prime} \omega_{i}\right\} .
\end{aligned}
$$

The molecular state $\Phi_{i}$ represents an electron configuration and is a solution of the stationary two center Dirac equation

$$
H \Phi_{i}=\omega_{i} \Phi_{i} .
$$

$\chi_{f}^{(-)}$is an analogous solution, but with the future boundary condition

$$
\begin{aligned}
\lim _{t \rightarrow+\infty} \chi_{f}^{(-)}(t)= & \lim _{t \rightarrow+\infty} \Phi_{f}(R(t)) \\
& \cdot \exp \left\{-i \int^{t} \mathrm{~d} t^{\prime} \omega_{f}\right\} .
\end{aligned}
$$


If we expand the dynamical solutions in terms of the stationary two center configurations we obtain

$$
\chi_{n}^{( \pm)}(t)=\sum_{m} a_{n m}^{( \pm)} \Phi_{m} \exp \left\{-i \int^{t} \mathrm{~d} t^{\prime} \omega_{m}\right\}
$$

with

$$
\lim _{t \rightarrow \mp \infty} a_{n m}^{( \pm)}=\delta_{n m}
$$

By inserting (B4) into the equation of motion (B2) the configuration amplitudes $a_{n m}^{( \pm)}$are seen to fulfill a set of coupled differential equations:

$$
\begin{aligned}
\dot{a}_{n m}^{( \pm)}= & -\sum_{l} a_{m l}^{( \pm)} \exp \left\{i \int^{t} \mathrm{~d}^{\prime} t\left(\omega_{m}-\omega_{l}\right)\right\} \\
& \cdot\left\langle\Phi_{m}\left|\partial_{t}\right| \Phi_{l}\right\rangle
\end{aligned}
$$

In the following we present the main steps how to derive (3.14) from the configuration formalism. We concentrate on the intensity given by the square of the radiation amplitude summed over all (final) states:

$$
\begin{aligned}
I(\boldsymbol{k}, \lambda)= & \sum_{f}\left|c_{\boldsymbol{k} \lambda}^{f i}\right|^{2} \\
= & \int^{f} \mathrm{~d} t \mathrm{~d} t^{\prime} \sum_{f}\left\langle\chi_{i}^{(+)}\left(t^{\prime}\right)\left|\boldsymbol{j} \boldsymbol{q}_{\boldsymbol{k} \lambda}\right| \chi_{f}^{(-)}\left(t^{\prime}\right)\right\rangle \\
& \cdot\left\langle\chi_{f}^{(-)}(t)\left|\boldsymbol{j} \boldsymbol{q}_{\boldsymbol{k} \lambda}^{*}\right| \chi_{i}^{(+)}(t)\right\rangle .
\end{aligned}
$$

The crucial step of the proof is to replace the wavefunctions with future boundary condition by those with past boundary condition. This substitution is based on the unitarity of the time evolution operator, whence

$$
\begin{aligned}
\sum_{f} \mid & \left.\chi_{f}^{(-)}\left(t^{\prime}\right)\right\rangle\left\langle\chi_{f}^{(-)}(t)\right| \\
& =U\left(t^{\prime}, \infty\right) \sum_{f}\left|\Phi_{f}\right\rangle\left\langle\Phi_{f}\right| U^{+}(t, \infty) \\
& =U\left(t^{\prime}, t\right) \\
& =U\left(t^{\prime},-\infty\right) \sum_{f}\left|\Phi_{f}\right\rangle\left\langle\Phi_{f}\right| U^{+}(t,-\infty) \\
& =\sum_{f}\left|\chi_{f}^{(+)}\left(t^{\prime}\right)\right\rangle\left\langle\chi_{f}^{(+)}(t)\right| .
\end{aligned}
$$

As mentioned the one-to-one correspondence between the $\Phi_{f}$ and $\chi_{f}$ states is correct for an adiabatic interaction. Then we find that it is possible to write

$$
I(\boldsymbol{k}, \lambda)=\sum_{f}\left|\tilde{c}_{\boldsymbol{k} \lambda}^{f i}\right|^{2}
$$

with

$$
\begin{aligned}
\tilde{c}_{\boldsymbol{k} \lambda}^{f i}= & -i \int \mathrm{d} t e^{i \omega t} \\
& \cdot\left\langle\chi_{f}^{(+)}(t)\left|\boldsymbol{j} \cdot \boldsymbol{q}_{\boldsymbol{k} \lambda}^{*}\right| \chi_{\boldsymbol{i}}^{(+)}(t)\right\rangle .
\end{aligned}
$$

$\tilde{c}_{\boldsymbol{k} \lambda}^{f i}$ itself has no physical interpretation but it leads to the same intensity if we sum over a complete set of configurations.

Regarding a system of fixed electron number $N$ we can express $\chi^{(+)}$as a Slater determinant of single-particle solutions $\Psi^{(+)}$of (B2), which are identical to the solutions (2.16):

$$
\chi_{l}^{(+)}(t)=\frac{1}{(N !)^{1 / 2}}\left|\begin{array}{ccc}
\Psi_{l_{1}}^{(+)}(1) & \ldots & \Psi_{l_{1}}^{(+)}(N) \\
\vdots & & \vdots \\
\Psi_{l_{N}}^{(+)}(1) & \ldots & \Psi_{l_{N}}^{(+)}(N)
\end{array}\right| .
$$

$\Psi_{l_{1}}^{(+)}(i)$ denotes the $i$-th electron in the state $j$ of the configuration $l$. To calculate the transition matrix elements

$$
M=\left\langle\chi_{f}^{(+)}(t)\left|\boldsymbol{j} \cdot \boldsymbol{q}_{\boldsymbol{k} \lambda}^{*}\right| \chi_{i}^{(+)}(t)\right\rangle
$$

we define the following matrices of wave functions:

$$
(F)_{l j}=\left\langle\Psi_{f_{l}}^{(+)}(j)\left|, \quad(I)_{l j}=\right| \Psi_{i_{l}}^{(+)}(j)\right\rangle .
$$

Furthermore we use the notation $A_{(n k)}$ for a matrix $A$ after the $n$-th row and $k$-th column have been eliminated. Employing the expansion of determinants we can write

$$
\begin{aligned}
M= & \frac{1}{N !} \sum_{n, m}^{N}(-)^{n+m}\left\langle\Psi_{f_{n}}^{(+)}\left|\boldsymbol{j} \cdot \boldsymbol{q}_{k \lambda}^{*}\right| \Psi_{i_{m}}^{(+)}\right\rangle \\
& \cdot \sum_{k=1}^{N}\left|F_{(n k)}\right|\left|I_{(m k)}\right|,
\end{aligned}
$$

where the multiplication of the two determinants involves taking the scalar product of the wave functions. This product can be re-expressed as

$$
\sum_{k}\left|F_{(n k)}\right|\left|I_{(m k)}\right|=N !\left|S_{(m n)}^{f i}\right|
$$

with

$$
\left(S^{f i}\right)_{k l}=\left\langle\Psi_{f e}^{(+)} \mid \Psi_{i_{k}}^{(+)}\right\rangle=\delta f_{l} i_{k} .
$$

At this point the meaning of the substitution $c_{\boldsymbol{k} \lambda}^{f i} \rightarrow \tilde{c}_{\boldsymbol{k} \lambda}^{f i}$ becomes clear. If we had worked with the radiation amplitude $c_{\boldsymbol{k} \lambda}^{f i}$ we would have formally obtained the same result but the matrix element

$$
\left(S^{f i}\right)_{k l}=\left\langle\Psi_{f_{e}}^{(-)} \mid \Psi_{i_{k}}^{(+)}\right\rangle
$$

would not be a Kronecker delta. Now it follows for $\tilde{c}_{\boldsymbol{k} \lambda}^{f i}$

$$
\tilde{c}_{\boldsymbol{k} \lambda}^{f i}=-i \sum_{n, m}^{N}(-)^{n+m}\left|S_{(m n)}^{f i}\right| H_{\boldsymbol{k} \lambda}^{f_{n} i_{m}}
$$

with

$$
H_{\boldsymbol{k} \lambda}^{f_{n} i_{m}}=\int \mathrm{d} t e^{i \omega t}\left\langle\Psi_{f_{n}}^{(+)}(t)\left|\boldsymbol{j} \cdot \boldsymbol{q}_{\boldsymbol{k} \lambda}^{*}\right| \Psi_{i_{m}}^{(+)}(t)\right\rangle .
$$

To evaluate the condition $\left(S^{\mathrm{fi}}\right)_{k l}=\delta_{f_{i} i_{k}}$ we investigate the final configurations systematically, 
which leads to three different cases:

(a) In the first case $f$ is identical to $i$. Then we have $\left|S_{(m n)}^{i i}\right|=\delta_{m n}$ and therefore

$$
\tilde{c}_{\boldsymbol{k} \lambda}^{i i}=-i \sum_{m \in i} H_{\boldsymbol{k} \lambda}^{m m}
$$

(b) The next possibility involves all configurations $f^{(1)}$ which differ from $i$ by one excited electron. Then $\left|S_{(m n)}^{f_{(1)} i}\right|$ is non-zero only for one pair $m$ and $n$, whence we have (up to a sign)

$$
\tilde{c}_{\boldsymbol{k} \lambda}^{f^{(1)} i} \sim i H_{\boldsymbol{k} \lambda}^{n m}, \quad n \notin i, \quad m \in i .
$$

(c) Since the rank of $S^{f i}$ is given by

$$
\operatorname{rk} S^{\mathrm{f} i}=N-N_{\mathrm{ex}} \text {, }
$$

[1] B. Müller, J. Rafelski, and W. Greiner, Z. Phys. 257, 62 (1972).

[2] J. Schwinger, Phys. Rev. 91, 713 (1953).

[3] P. Roman, Introduction to Quantum Field Theory, John Wiley \& Cie, New York (1969.

[4] T. H. Rihan, N. S. Aly, E. Merzbacher, B. Müller, and W. Greiner, Z. Phys. A 285, 397 (1978).

[5] B. Müller and W. Greiner, Z. Naturforsch. 31 a, 1 (1976).

[6] W. R. Thorson and J. B. Delos, Phys. Rev. A 18, 117 (1978).

[7] J. F. Reading and A. L. Ford, Phys. Rev. A21,124 (1980).

[8] B. Müller and W. Greiner, Phys. Rev. Lett. 33, 469 (1974).

[9] J. S. Greenberg, C. K. Davis, and P. Vincent, Phys. Rev. Lett. 33, 473 (1974). where $N_{\text {ex }}$ is the number of excited electrons, $\left|S_{(m n)}^{\mathrm{fi}}\right|$ vanishes for all configurations with $N_{\mathrm{ex}} \geqq 2$ because of $r k S_{(m n)}^{\mathrm{f} i} \leqq r k S^{\mathrm{f} i}<N-1$.

Concluding these results we obtain for the intensity

$$
I(\boldsymbol{k}, \lambda)=\left|\tilde{c}_{\boldsymbol{k} \lambda}^{i i}\right|^{2}+\sum_{f(1)}\left|\tilde{c}_{\boldsymbol{k} \lambda}^{f^{(1)} i}\right|^{2},
$$

which leads to (3.14). Note that

$$
\left|\tilde{c}_{\boldsymbol{k} \lambda}^{i i}\right|^{2}=\left|\sum_{n}^{\mathrm{HF}} H_{k \lambda}^{n n}\right|^{2}
$$

because of the fixed number of electrons.

[10] B. Müller, R. K. Smith, and W. Greiner, Phys. Lett. 53B, 401 (1975).

[11] R. Anholt, Z. Phys. A 288, 257 (1978).

[12] J. S. Briggs and K. Dettmann, J. Phys. B 10, 1113 (1977).

[13] W. Wölfli, Ch. Stoller, G. Bonani, M. Stöckli, M. Suter, and W. Däppen, Z. Phys. A 286, 249 (1978).

[14] Ch. Stoller, W. Wölfli, G. Bonani, M. Stöckli, and M. Suter, J. Phys. B 10, L347 (1977); Ch. Stoller, W. Wölfli, G. Bonani, E. Morenzoni, and M. Stöckli, Z. Phys. A 287, 33 (1978).

[15] M. Gros, P. T. Greenland, and W. Greiner, Z. Phys. A 280, 31 (1977).

[16] K. Bleuler, Helv. Phys. Acta 23, 567 (1950); S. N. Gupta, Proc. Phys. Soc. London A 63, 681 (1950). 